\title{
Acute Inhibition of PKA Activity at Old Ages Ameliorates Age-Related Memory Impairment in Drosophila
}

\author{
Daisuke Yamazaki, ${ }^{1,2}$ Junjiro Horiuchi, ${ }^{2,3}$ Tomoyuki Miyashita, ${ }^{1}$ and Minoru Saitoe ${ }^{1}$ \\ ${ }^{1}$ Tokyo Metropolitan Institute for Neuroscience, Fuchu, Tokyo 183-8526, Japan, ${ }^{2}$ Laboratory of Cellular Genetics, and ${ }^{3}$ Center for Priority Areas, Tokyo \\ Metropolitan University, Tokyo 192-0397, Japan
}

Age-related memory impairment (AMI) is a critical and debilitating phenotype of brain aging, but its underlying molecular mechanisms are largely unknown. In Drosophila, AMI is highly correlated with PKA activity in the mushroom bodies, neural centers essential for forming associative olfactory memories. Heterozygous mutations in $D C O(D C 0 /+)$, which encodes the major catalytic subunit of PKA (PKAc), significantly suppress AMI, while overexpression of a $D C 0$ transgene $\left(D C 0^{+}\right)$impairs memory and occludes AMI. PKA activity does not increase upon aging, and it is not clear whether AMI is caused by continual PKA activity throughout aging or by an acute increase in PKA signaling at old ages. Likewise, it is not clear whether AMI can be ameliorated by acute interventions at old ages or whether continuous intervention throughout aging is necessary. We show here that an acute increase in PKA activity at old ages is sufficient to restore normal AMI in $\mathrm{DCO} /+$ flies. Conversely, acute expression of a PKA inhibitory peptide at old ages is sufficient to reverse AMI in a wild-type background. These results indicate that AMI in Drosophila is caused by an age-dependent change in PKA-dependent signaling that can be reversed by acute interventions at old ages.

\section{Introduction}

Aging has often been proposed to result from a gradual, irreversible accumulation of deleterious byproducts over time. For example, accumulation of oxidative damage may cause age-related cellular damage and degeneration (Wallace and Melov, 1998; Golden et al., 2002; Balaban et al., 2005). If this is the case, interventions that delay aging may require long-term treatments throughout the lifespan of the organism. On the other hand, there is also evidence that mortality rates can be influenced by acute dietary interventions, suggesting that interventions specifically at old ages can influence age-related phenotypes (Mair et al., 2003).

Age-related memory impairment (AMI) is a critical and debilitating phenotype of brain aging, but its underlying mechanisms are still largely unknown. In Drosophila, which has an average lifespan of 30-40 d after eclosion, AMI of an aversive olfactory association becomes significant at $15 \mathrm{~d}$ of age and consists of a specific and progressive decline in middle-term memory (MTM), which can be measured as a decrease in memory, $1 \mathrm{~h}$ after training ( $1 \mathrm{~h}$ memory) (Tamura et al., 2003). AMI is strongly suppressed in heterozygous $\mathrm{DCO} /+$ mutants, which are

Received June 22, 2010; revised Sept. 9, 2010; accepted Sept. 21, 2010.

This work was supported by a Grant-in-Aid on Innovative Areas "Systems Molecular Ethology" and a grant for Scientific Research (B) (21300144) from the Ministry of Education, Culture, Sports, Science, and Technology to M.S. D.Y. is supported by a fellowship from the Japan Society for the Promotion of Science. We thank Drs. Haruo Okado and Chiaki Maruyama for critical reading of the manuscript and members of the Saitoe laboratory for their assistance and discussions.

Correspondence should be addressed to Minoru Saitoe, Tokyo Metropolitan Institute for Neuroscience, 2-6 Musashi-dai, Fuchu, Tokyo 183-8526, Japan. E-mail: saito-mn@igakuken.or.jp.

D. Yamazaki's present address: Laboratory of Morphogenesis, Institute of Molecular and Cellular Biosciences, University of Tokyo, Yayoi 1-1-1 Bunkyo-ku, Tokyo 113-0032, Japan.

DOI:10.1523/JNEUROSCI.3229-10.2010

Copyright $\odot 2010$ the authors $\quad$ 0270-6474/10/3015573-05\$15.00/0 defective for DCO (Yamazaki et al., 2007), the gene encoding the major catalytic subunit of protein kinase A. Decreasing PKA to $\sim 50 \%$ of wild-type activity delays AMI onset to over $30 \mathrm{~d}$ and significantly decreases its severity. AMI can be restored in $\mathrm{DCO} /+$ flies by expressing a $D C O$ transgene in the mushroom bodies (MBs), brain structures important for olfactory associative memory in insects.

Memory in Drosophila is highly dependent on PKA activity in the MBs (McGuire et al., 2005). Large decreases in PKA activity, to $\sim 20 \%$ of the wild-type amount (Drain et al., 1991; Skoulakis et al., 1993; Li et al., 1996; Goodwin et al., 1997), as well as increases in PKA activity (Drain et al., 1991), inhibit memory, while the milder twofold reductions in activity seen in $\mathrm{DCO} /+$ flies improve a consolidated form of memory in young flies (Horiuchi et al., 2008) and delay AMI in old flies (Yamazaki et al., 2007). However, neither DCO expression nor PKA activity increases upon aging (Yamazaki et al., 2007). These data suggest that AMI in Drosophila may be caused either by accumulation of damage produced by chronic cAMP/PKA activity throughout aging or through an acute, deleterious, change in activity of a signaling pathway upon old age that occurs downstream of PKA activity. To test this idea, we altered PKA amounts in the MBs in a temporal fashion using the RU486-inducible gene-switch system (Osterwalder et al., 2001) to determine when PKA activity causes AMI, and when activity needs to be inhibited to ameliorate AMI.

\section{Materials and Methods}

Fly stocks. The wild-type control line, $w(C S 10)$ has been described before (Yamazaki et al., 2007). UAS-PKAc F5.9 and UAS-PKI F12-2 (Kiger et al., 1999), provided by J. A. Kiger, Jr. (University of California Davis, Davis, CA) were used and are referred to as $U A S-D C 0^{+}$and $U A S-P K I$ in this manuscript. The $P\{M B-S w i t c h\} 12-1$ line, referred to as MBS, was pro- 

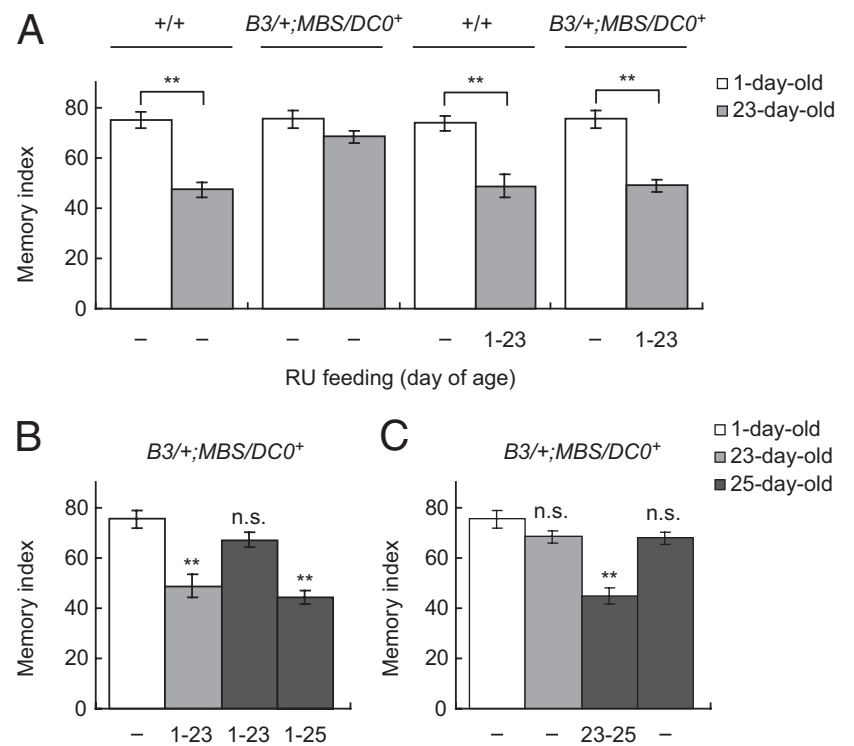

$\mathrm{RU}$ feeding (day of age)

$\mathrm{RU}$ feeding (day of age)

Figure 1. Acute expression of a $D C O$ transgene $\left(D C O^{+}\right)$in the mushroom bodies at old age restores AMI in DCO/+ mutants. A, Flies were either fed food containing RU486 continuously from 1 to $23 \mathrm{~d}$ of age (1-23) or fed food lacking RU486 (-). Memory was tested at 1 or $23 \mathrm{~d}$ of age. Continuous RU486 feeding restores $\mathrm{AMl}$ in aged $B 3 /+; M B S / D C O^{+}$flies. $N=6-10$. $B$, Flies were fed food containing RU486 from 1 to $23 \mathrm{~d}$ or 1 to $25 \mathrm{~d}$ or fed food lacking RU486. Memory was tested at 1,23 , or $25 \mathrm{~d}$ of age. Memory improves to young levels in $B 3 /+; M B S / D C O^{+}$flies when RU486 feeding is stopped $2 \mathrm{~d}$ before measuring memory. $N=8-10$. C, Flies were fed food containing RU486 from 23 to $25 \mathrm{~d}$ of age (23-35) or fed food without RU486. Feeding RU486 specifically at old age restored AMI in $B 3 /+; M B S / D C O^{+}$flies. $N=8-10$. All data are expressed as means $\pm S E M$. Data from each panel were analyzed by one-way ANOVA followed by Tukey's multiple-comparison tests. Differences between young and aged or aged, RU486treated flies are indicated. n.s., No significant difference; ${ }^{* *} p<0.05$.

vided by R. Davis. Fly stocks were maintained at $25 \pm 2{ }^{\circ} \mathrm{C}$ and $60 \pm 10 \%$ relative humidity under a $12 / 12 \mathrm{~h}$ light/dark cycle.

Memory assay. Standard single-cycle aversive olfactory conditioning was performed as previously described (Tully and Quinn, 1985; Yamazaki et al., 2007). About 100 flies were exposed sequentially to two aversive odors (3-octanol and 4-methylcyclohexanol) for $60 \mathrm{~s}$ with $45 \mathrm{~s}$ rest intervals after each odor presentation. During exposure to the first (CS+) odor, flies were also subjected to the unconditioned stimulus, $1.5 \mathrm{~s}$ pulses of $60 \mathrm{~V}$ DC electric shocks, every $5 \mathrm{~s}$. The second odor (CS-) was not paired with shocks. To test for memory retention, trained flies were placed at the choice point of a T-maze and allowed to choose from the CS + and CS - . Memory was measured as a memory index (Tully and Quinn, 1985) such that a 50:50 distribution (no memory) yielded a memory index of 0 and a 0:100 distribution away from the CS+ yielded a memory index of 100 . For memory retention curves, different groups of flies were trained and tested for each time point.

Sensorimotor responses to odors and electrical shocks. Peripheral control experiments including avoidance of odors and electrical shocks were performed as previously described (Tully and Quinn, 1985). Approximately 100 naive flies were placed at a choice point of a T-maze, where they had to choose between an odor (OCT or MCH) and mineral oil (odor avoidance) or between electrically shocked and nonshocked conditions (shock avoidance). An avoidance index was calculated as described for memory assays.

RU486 treatment. RU486 was dissolved in ethanol at various concentrations and mixed with fly food to a final concentration of $4 \%$ ethanol (Osterwalder et al., 2001; Mao et al., 2004). Effective concentrations of RU486 were determined by measuring PKI transcripts in UAS-PKI;;MBSwitch (PKI;;MBS) flies fed food containing various concentrations of RU486 (data not shown). For memory assays, flies were returned to normal food $2 \mathrm{~h}$ before training to avoid nonspecific inhibitory effects of RU486 on memory (Mao et al., 2004).

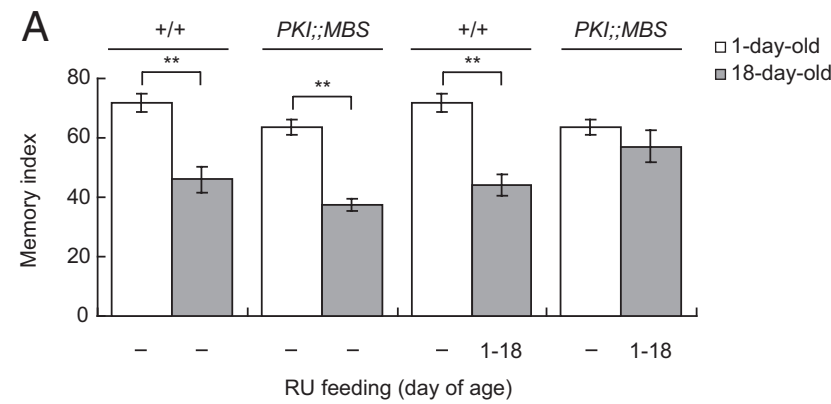

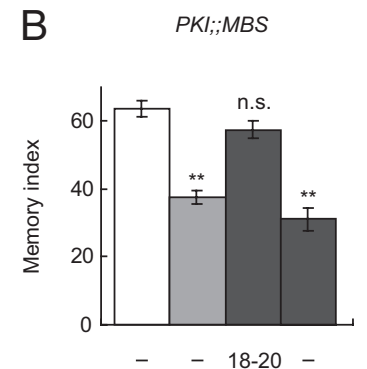

$\mathrm{RU}$ feeding (day of age)
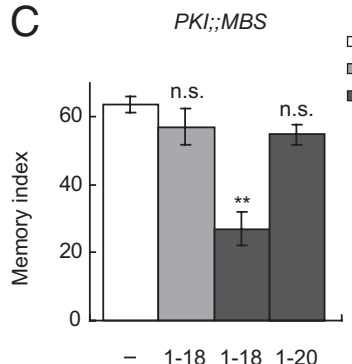

$\mathrm{RU}$ feeding (day of age)
Figure 2. Acute expression of the PKA inhibitor, PKL, in the mushroom bodies at old age suppresses AMI. A, Flies were fed RU486 from 1 to $18 \mathrm{~d}$ of age (1-18) or fed without RU486 (-). Memory was tested at 1 or $18 \mathrm{~d}$ of age. Continuous RU486 feeding suppresses AMI in PKI;; MBS flies. $N=6-12$. $B$, Flies were fed RU 486 from 18 to $20 \mathrm{~d}$ of age $(18-20)$ or fed without RU486 and memory was tested at 1,18 , or $20 \mathrm{~d}$ of age. Acute feeding of RU486 at old age suppresses AMl in PKl;;MBS flies. $N=8-12$. C, Flies were fed RU486 from 1 to 18 or 1 to $20 \mathrm{~d}$ of age or fed without RU486 and memory was tested at 1, 18, or $20 \mathrm{~d}$ of age. AMl is restored in 20-d-old PKI;;MBS flies when RU486 feeding is stopped at $18 \mathrm{~d}$ of age. $N=7-12$. All data are expressed as means \pm SEM. Data were analyzed as described in the legend to Figure 1.

Statistics. Statistical analyses were performed using Prism version 4.01 (GraphPad). $p$ values $<0.05$ were judged as statistically significant.

\section{Results}

Olfactory associative memory is tightly regulated by PKA activity in the MBs. Thus, expressing a $D C 0$ transgene $\left(D^{\circ} O^{+}\right)$in the MBs inhibits $1 \mathrm{~h}$ memory in a wild-type background, while it restores normal AMI in a DCO/+ background, which has decreased basal PKA activity (Yamazaki et al., 2007). In MB-Switch/UAS-DCO ${ }^{+}$ $\left(\mathrm{MBS} / \mathrm{DCO}^{+}\right)$flies, a $\mathrm{DCO}$ transgene can be induced in the MBs by feeding flies RU486. However, we were unable to detect a specific increase in DCO transcripts from head extracts of $M B S / D C O^{+}$ flies, likely due to a low signal-to-noise ratio (data not shown). Therefore, to determine the appropriate RU486 treatment regimen for induction of the $D C 0$ transgene and clearance of induced $D C 0$ gene product, we measured $1 \mathrm{~h}$ memory after aversive olfactory conditioning. We determined that feeding $1 \mathrm{mM}$ RU486 for $48 \mathrm{~h}$ was sufficient to inhibit $1 \mathrm{~h}$ memory in a wild-type background, while it had no effect on $1 \mathrm{~h}$ memory in young flies from a $D C 0 /+$ background, $D C 0^{B 3} /+; M B-S w i t c h / U A S-D C 0^{+}(B 3 /+$; $M B S / D C O^{+}$) (supplemental Fig. $1 A$, available at www.jneurosci. org as supplemental material). Returning flies to normal food for $48 \mathrm{~h}$ after RU486 feeding was sufficient to restore normal memory in a wild-type background (supplemental Fig. $1 B$, available at www.jneurosci.org as supplemental material). This indicates that inhibition of memory upon PKA overexpression is an acute effect that can be rescued by restoring normal PKA activity.

AMI was suppressed at $23 \mathrm{~d}$ of age in $\mathrm{B3} /+; \mathrm{MBS} / \mathrm{DCO}^{+}$flies raised in the absence of RU486 (Fig. $1 A$ ), indicating that the $D C 0$ transgene is not expressed at levels sufficient to restore AMI in a DCO/+ background when RU486 is lacking. On the other hand, 

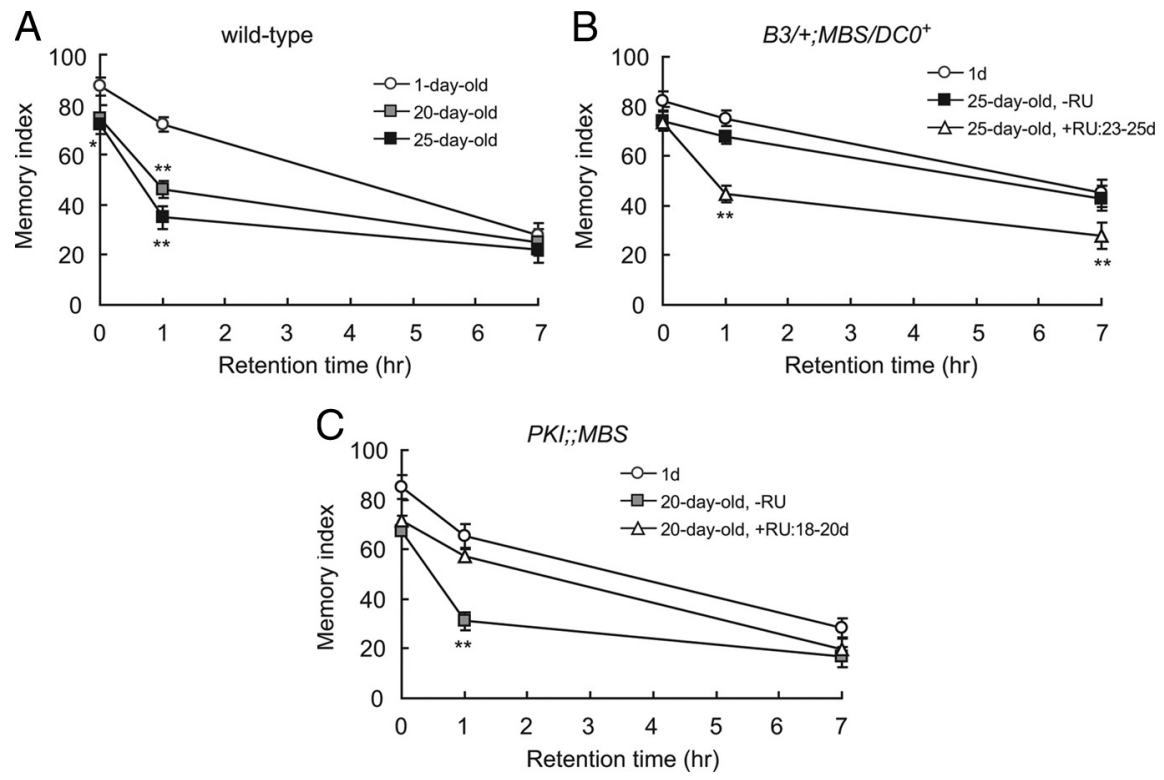

Figure 3. Acute changes in PKA activity at old ages shifts memory retention curves between young and old states. A, Wild-type flies show characteristic changes in the shape of their memory retention curves upon aging. One hour memory is severely reduced, while 3 min memory $(0 \mathrm{~h})$ and $7 \mathrm{~h}$ memory are only mildly affected. Two-way ANOVA indicates significant differences in memory due to retention time $(p<0.0001)$, age $(p<0.0001)$, and interaction between age and retention time $(p=0.0011)$. Bonferroni post hoc analyses demonstrate a slight but significant decrease in 3 min memory between 1-d-old and 25 -d-old flies $\left({ }^{*} p<0.05\right)$ and a larger decrease in $1 \mathrm{~h}$ memory between 1-d-old and 20-d-old $\left({ }^{* *} p<0.001\right)$ and 25 -d-old $\left({ }^{* *} p<0.001\right)$ flies. $B$, Flies from a $D C 0^{B 3} /+$ background do not show AMI and maintain youthful memory up to $25 \mathrm{~d}$ of age. Acute induction of a $D C O$ transgene in $\mathrm{B3} /+; \mathrm{MBS} / \mathrm{DCO} \mathrm{O}^{+}$flies (feeding RU486 from 23 to $25 \mathrm{~d}$ of age) shifts the retention curve to that of old wild-type flies. Two-way ANOVA indicates significant differences due to retention time $(p<0.0001)$, age and drug treatment $(p<0.0001)$, and the interaction between retention time and age and drug treatment $(p=0.0041)$. Bonferroni post hoc analyses do not demonstrate any significant differences in memory between 1-d-old and 25 - $d$-old $B 3 /+; M B S / D C O^{+}$non-drug-treated flies, but they do demonstrate significant changes in $1 \mathrm{~h}$ and $7 \mathrm{~h}$ memory upon acute treatment with RU486 ${ }^{* *} p<0.001$ in both cases comparing 25-d-old RU486-treated flies to both 1-d-old and 25-d-old non-drug-treated flies). C, PKl;;MBS flies show normal AMI in the absence of RU486 feeding. Acute inhibition of PKA by feeding RU486 at 18-20 d of age suppresses AMI and shifts the memory retention curve to that of young flies. Two-way ANOVA indicates significant differences due to retention time $(p<0.0001)$, age and drug treatment $(p<0.0001)$, and the interaction between retention time and age and drug treatment $(p=0.0139)$. Bonferroni post hoc analyses demonstrate a significant difference in $1 \mathrm{~h}$ memory between 1-d-old and non-drug-treated 20-d-old PKI;;MBS flies (** $p<0.001)$. This difference is abolished when PKI;; MBS flies are acutely fed RU486 from 18 to $20 \mathrm{~d}$ of age. $N=$ $8-12$ for all data.

we observed $\mathrm{AMI}$ in $B 3 /+; M B S / D C 0^{+}$flies continuously fed RU486 from 1 to $23 \mathrm{~d}$ of age (Fig. $1 \mathrm{~A}$ ), confirming that continual PKA expression during aging causes AMI.

To examine the temporal effect of $D C 0$ transgene expression on AMI during aging, we fed $B 3 /+; M B S / D C 0^{+}$flies RU486 from 1 to $23 \mathrm{~d}$ of age, switched the food to normal food for $2 \mathrm{~d}$, and then tested memory at $25 \mathrm{~d}$ of age. Although significant AMI was observed in 23 -d-old $B 3 /+; M B S / D C 0^{+}$flies fed RU486 from 1 to $23 \mathrm{~d}$ of age and 25 -d-old $B 3 /+; M B S / D C 0^{+}$flies fed RU486 from 1 to $25 \mathrm{~d}$ of age, AMI was completely suppressed in 25 -d-old flies that were fed food lacking RU486 for the last $2 \mathrm{~d}$ (Fig. $1 B$ ). Conversely, although AMI did not occur in 25-d-old $B 3 /+; M B S / D C 0^{+}$ flies fed normal food, we observed a decrease in $1 \mathrm{~h}$ memory when these flies were fed RU486 from 23 to $25 \mathrm{~d}$ of age (Fig. 1C). Importantly, since induction of the $D C 0$ transgene at young ages (feeding RU486 from 1 to $3 \mathrm{~d}$ of age) did not affect $1 \mathrm{~h}$ memory in 3 -d-old $\mathrm{B} 3 /+; \mathrm{MBS} / \mathrm{DCO}^{+}$flies (supplemental Fig. $2 \mathrm{~A}$, available at www.jneurosci.org as supplemental material; Fig. $1 A$ ), these effects are age specific and likely associated with AMI. In addition, RU486 treatment throughout aging did not restore AMI in control $B 3 /+; M B S /+$ and $B 3 /+; D C 0^{+}$flies (supplemental Fig. $2 B$, available at www.jneurosci.org as supplemental material) and did not affect task-related odor and shock responses in B3/
$+; M B S / D C 0^{+}$flies (supplemental Fig. $2 C$, available at www.jneurosci.org as supplemental material). Thus, our observed effects are due to PKA expression rather than to nonspecific effects of genetic background, RU486 feeding, or altered sensorimotor responses. Together, our results suggest that AMI does not result from accumulation of damage caused by chronic PKA activity throughout aging, but rather results from an increase in PKA-dependent signaling specifically at old ages.

If AMI is caused by an increase in PKA-dependent signaling, it should be ameliorated in a wild-type background by acutely inhibiting PKA at old ages. To test this idea, we expressed PKI, a competitive peptide inhibitor of PKA (Drain et al., 1991; Kiger et al., 1999), in the MBs in UAS-PKI;;MB-Switch (PKI;;MBS) flies at various periods during aging. We previously reported that feeding $1 \mathrm{~mm}$ RU486 to young (1-3 d old) PKI;;MBS flies does not affect $1 \mathrm{~h}$ memory (Yamazaki et al., 2007). However, continuous feeding of RU486 during aging, from 1 to $18 \mathrm{~d}$ of age, significantly suppressed AMI at $18 \mathrm{~d}$ of age compared to 18-d-old wild-type flies and PKI;MBS flies raised in the absence of RU486 (Fig. 2A). Thus, the amount of PKI induced by $1 \mathrm{~mm}$ RU486 is sufficient to suppress AMI. When PKI;;MBS flies were fed RU486 from 18 to $20 \mathrm{~d}$ of age, $1 \mathrm{~h}$ memory at $20 \mathrm{~d}$ was significantly higher than in control flies that were not fed RU486 (Fig. 2 B). Conversely, we observed significant AMI in 20-d-old PKI;;MBS flies fed RU486 from 1 to $18 \mathrm{~d}$ of age and returned to normal food from days 18 to 20 (Fig. 2C). RU486 treatment did not suppress AMI in transgenic control MBS and PKI flies (supplemental Fig. $3 A$, available at www.jneurosci.org as supplemental material) and did not affect task-related odor and shock responses in PKI;;MBS flies (supplemental Fig. 3B, available at www.jneurosci.org as supplemental material). These results are consistent with a model where acute PKA activity in aged flies causes AMI.

Our data demonstrate that $1 \mathrm{~h}$ memory in old flies can be improved to young levels by an acute, mild reduction in PKA activity. This reduction has no effect on memory in young flies, suggesting that aging causes increases in PKA-dependent signaling, which inhibits memory. To further support our hypothesis that these effects of PKA are related to AMI, we generated and compared memory retention curves of young and old flies having different PKA activities. AMI causes very specific, reproducible changes in memory retention curves in wild-type flies (Tamura et al., 2003). One-hour memory is severely reduced, while $3 \mathrm{~min}$ memory, sometimes referred to as initial learning, and $7 \mathrm{~h} \mathrm{mem-}$ ory are unaffected or only mildly affected (Fig. $3 A$ ). Memory retention curves of aged flies are indistinguishable from curves of amnesiac memory mutants (Feany and Quinn, 1995), which are defective specifically for MTM (DeZazzo et al., 1999). amnesiac flies do not show changes in memory upon aging, indicating that 
MTM is the major memory phase affected by AMI after singlecycle olfactory training (Tamura et al., 2003). In DCO/+ flies, memory does not change at any retention time upon aging to $20 \mathrm{~d}$, and memory retention curves of old $\mathrm{DCO} /+$ flies are reminiscent of those of young wild-type flies (Yamazaki et al., 2007).

When a DCO transgene was expressed acutely in the MBs of aged $\mathrm{DCO} /+$ flies, the memory retention curve shifted from one resembling that of young wild-type flies to a curve indistinguishable from curves of old wild-type flies (Fig. $3 A, B$ ). Three-minute memory was unaffected, while $1 \mathrm{~h}$ memory was reduced to levels seen in aged wild-type flies. Similar to previously reported $B 3 /+$ flies (Horiuchi et al., 2008), B3/+;MBS/DCO${ }^{+}$flies had increased $7 \mathrm{~h}$ memory compared to wild-type flies, but this is an effect on anesthesia-resistant memory, a long-lasting memory produced by single-cycle training, that is unrelated to MTM and AMI. Upon acute induction of a DCO transgene, $7 \mathrm{~h}$ memory was also reduced to wild-type levels.

In PKI; MBS flies, AMI occurs normally in the absence of RU486 feeding and memory retention curves of young and old flies were indistinguishable from those of corresponding wildtype flies (Fig. $3 A, C$ ). When RU486 was fed to these flies specifically at old age, 3 min memory and $7 \mathrm{~h}$ memory were unaffected, while a clear rescue of $1 \mathrm{~h}$ memory was observed (Fig. $3 C$ ). These data demonstrate that an increase in PKA activity at old ages predominantly affects MTM, the memory phase affected by AMI.

\section{Discussion}

AMI has often been proposed to be an irreversible effect caused by accumulation of deleterious products that cause neuronal dysfunction. However, our current results suggest that there is an acute and reversible component of AMI that depends on PKA activity in the MBs. While decreases in PKA activity to $~ 50 \%$ of wild-type activity significantly delay AMI, PKA activity itself does not change upon aging (Yamazaki et al., 2007). Thus, we first hypothesized that continual PKA activity during aging results in accumulation of products that cause AMI. However, acute overexpression of PKA in the MBs at old ages is sufficient to restore $\mathrm{AMI}$ in $\mathrm{DCO} /+$ flies, while acute expression of a PKA inhibitory peptide in the MBs at old ages significantly ameliorates AMI in wild-type flies. These results suggest that while PKA activity per se does not increase upon aging, an age-related increase in PKAdependent signaling, likely at a step downstream of PKA itself, contributes to AMI.

The PKA-dependent component that disrupts memory formation upon aging is still not known. Increased PKA-mediated phosphorylation of L-type voltage-gated calcium channels (LVGCCs) and tau, a major component of neurofibrillary tangles (NFTs), is suspected to disrupt neuronal functions required for memory formation (Morrison and Hof, 1997; Foster, 2002; Davare and Hell, 2003; Liu et al., 2004). Treatment with the LVGCC inhibitor nimodipine preferentially improves learning and memory in aged rabbits and rats (Deyo et al., 1989; Levere and Walker, 1992), indicating that an age-related increase in LVGCC activity through PKA-mediated phosphorylation may be a cause of AMI. Accumulation of NFTs shows a strong correlation with AMI (Morrison and Hof, 1997), and PKA is one of several kinases that phosphorylate tau protein and facilitate NFT formation (Liu et al., 2004). However, we have not found any age-related increases in expression of LVGCCs or tau in Drosophila (T. Miyashita, unpublished observation).

Even if both PKA activity and expression of PKA substrates do not increase, PKA-dependent signaling can be enhanced by inhibiting dephosphorylation or degradation of phosphorylated substrates. Interestingly, we observed decreases in amounts of $\mathrm{A} 1, \mathrm{~B}$, and $\mathrm{B} 2$ subunits of calcineurin, $\mathrm{a} \mathrm{Ca}^{2+}$-dependent protein phosphatase, in aged flies (T. Miyashita, unpublished observations). Furthermore, we also observed age-related decreases in expression of a regulatory subunit of PKA, Pka-R1 (T. Miyashita, unpublished observation). Age-related changes in amounts of these phosphatases and regulatory subunits may contribute an age-related increase in PKA-dependent signaling.

AMI is likely to be a complex phenomenon with many different causes. Although it is strongly suppressed in $\mathrm{DCO} /+$ mutants, $\mathrm{DCO} /+$ flies eventually show AMI at $\sim 30 \mathrm{~d}$ of age (Yamazaki et al., 2007). Therefore, there may be a component of AMI, which is independent of PKA activity. In this paper, we suggest that PKAdependent AMI is likely due to acute changes at old age in activity of a PKA-dependent signaling pathway required for MTM formation. We propose that there is an optimal window of activity of this putative pathway that is necessary for maximal MTM formation. As flies age, activity of this pathway changes, leading to decreased MTM and AMI. Acute decreases in PKA activity suppress these age-dependent changes, shifting pathway activity back toward maximal MTM formation. Thus expressing PKA restores $\mathrm{AMI}$ in a $\mathrm{DCO} /+$ background while inhibiting PKA ameliorates AMI in a wild-type background.

In wild-type flies, AMI is first observed at $15 \mathrm{~d}$ of age but becomes progressively more severe upon further aging. In $D C 0$ heterozygotes, AMI does not occur until $30 \mathrm{~d}$ of age. However, similar to other aging phenotypes including lifespan, the progression and severity of AMI are influenced by environmental factors, including seasonal fluctuations in temperature and humidity, even in a controlled laboratory environment. Thus, in all our experiments, we specifically chose fly ages where robust AMI (a decrease in $1 \mathrm{~h}$ memory of just over 20 PI units) occurs in wildtype flies while not in $B 3 /+$ flies (no significant decrease in $1 \mathrm{~h}$ memory). These fly ages corresponded to $23-25 \mathrm{~d}$ for $B 3 /+; M B S /$ $\mathrm{DCO}^{+}$experiments and $18-20 \mathrm{~d}$ for PKI;;MBS experiments.

In mammals, inhibition of PKA activity in the prefrontal cortex also improves working memory in aged, cognitively impaired rats but not in young rats (Ramos et al., 2003). Similar to Drosophila MTM, working memory is a transient form of memory. These data indicate that there may be a conserved PKA-dependent signaling whose activity increases upon aging and impairs a short form of memory in both vertebrate and invertebrates. Recently, it has been reported that aging also affects long-term memory, a consolidated type of aversive memory that is formed only after multiple trainings with interspersed rest intervals (Mery, 2007). While it will be of interest in the future to determine whether defects in long-term memory can also be ameliorated by acute treatments, our current observations provide new insights into developing strategies to combat short-term cognitive declines that occur upon aging.

\section{References}

Balaban RS, Nemoto S, Finkel T (2005) Mitochondria, oxidants, and aging. Cell 120:483-495.

Davare MA, Hell JW (2003) Increased phosphorylation of the neuronal L-type $\mathrm{Ca}(2+)$ channel $\mathrm{Ca}(\mathrm{v}) 1.2$ during aging. Proc Natl Acad Sci U S A 100:16018-16023.

Deyo RA, Straube KT, Disterhoft JF (1989) Nimodipine facilitates associative learning in aging rabbits. Science 243:809-811.

DeZazzo J, Xia S, Christensen J, Velinzon K, Tully T (1999) Developmental expression of an $\mathrm{amn}(+)$ transgene rescues the mutant memory defect of amnesiac adults. J Neurosci 19:8740-8746.

Drain P, Folkers E, Quinn WG (1991) cAMP-dependent protein kinase and the disruption of learning in transgenic flies. Neuron 6:71-82. 
Feany MB, Quinn WG (1995) A neuropeptide gene defined by the Drosophila memory mutant amnesiac. Science 268:869-873.

Foster TC (2002) Regulation of synaptic plasticity in memory and memory decline with aging. Prog Brain Res 138:283-303.

Golden TR, Hinerfeld DA, Melov S (2002) Oxidative stress and aging: beyond correlation. Aging Cell 1:117-123.

Goodwin SF, Del Vecchio M, Velinzon K, Hogel C, Russell SR, Tully T, Kaiser K (1997) Defective learning in mutants of the Drosophila gene for a regulatory subunit of cAMP-dependent protein kinase. J Neurosci 17: 8817-8827.

Horiuchi J, Yamazaki D, Naganos S, Aigaki T, Saitoe M (2008) Protein kinase A inhibits a consolidated form of memory in Drosophila. Proc Natl Acad Sci U S A 105:20976-20981.

Kiger JA Jr, Eklund JL, Younger SH, O’Kane CJ (1999) Transgenic inhibitors identify two roles for protein kinase A in Drosophila development. Genetics 152:281-290.

Levere TE, Walker A (1992) Old age and cognition: enhancement of recent memory in aged rats by the calcium channel blocker nimodipine. Neurobiol Aging 13:63-66.

Li W, Tully T, Kalderon D (1996) Effects of a conditional Drosophila PKA mutant on olfactory learning and memory. Learn Mem 2:320-333.

Liu SJ, Zhang JY, Li HL, Fang ZY, Wang Q, Deng HM, Gong CX, GrundkeIqbal I, Iqbal K, Wang JZ (2004) Tau becomes a more favorable substrate for GSK-3 when it is prephosphorylated by PKA in rat brain. J Biol Chem 279:50078-50088.

Mair W, Goymer P, Pletcher SD, Partridge L (2003) Demography of dietary restriction and death in Drosophila. Science 301:1731-1733.

Mao Z, Roman G, Zong L, Davis RL (2004) Pharmacogenetic rescue in time and space of the rutabaga memory impairment by using Gene-Switch. Proc Natl Acad Sci U S A 101:198-203.

McGuire SE, Deshazer M, Davis RL (2005) Thirty years of olfactory learning and memory research in Drosophila melanogaster. Prog Neurobiol 76: $328-347$.

Mery F (2007) Aging and its differential effects on consolidated memory forms in Drosophila. Exp Gerontol 42:99-101.

Morrison JH, Hof PR (1997) Life and death of neurons in the aging brain. Science 278:412-419.

Osterwalder T, Yoon KS, White BH, Keshishian H (2001) A conditional tissue-specific transgene expression system using inducible GAL4. Proc Natl Acad Sci U S A 98:12596-12601.

Ramos BP, Birnbaum SG, Lindenmayer I, Newton SS, Duman RS, Arnsten AF (2003) Dysregulation of protein kinase a signaling in the aged prefrontal cortex: new strategy for treating age-related cognitive decline. Neuron 40:835-845.

Skoulakis EM, Kalderon D, Davis RL (1993) Preferential expression in mushroom bodies of the catalytic subunit of protein kinase A and its role in learning and memory. Neuron 11:197-208.

Tamura T, Chiang AS, Ito N, Liu HP, Horiuchi J, Tully T, Saitoe M (2003) Aging specifically impairs amnesiac-dependent memory in Drosophila. Neuron 40:1003-1011.

Tully T, Quinn WG (1985) Classical conditioning and retention in normal and mutant Drosophila melanogaster. J Comp Physiol A 157:263-277.

Wallace DC, Melov S (1998) Radicals r'aging. Nat Genet 19:105-106.

Yamazaki D, Horiuchi J, Nakagami Y, Nagano S, Tamura T, Saitoe M (2007) The Drosophila DCO mutation suppresses age-related memory impairment without affecting lifespan. Nat Neurosci 10:478-484. 